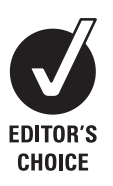

CHOICE

\title{
Building national estimates of the burden of road traffic injuries in developing countries from all available data sources: Iran
}

\author{
K Bhalla, ${ }^{1}$ M Naghavi, ${ }^{2}$ S Shahraz, ${ }^{1}$ D Bartels, ${ }^{1}$ C J L Murray ${ }^{2}$
}

${ }^{1}$ Harvard University Initiative for Global Health, Cambridge, Massachusetts, USA; ${ }^{2}$ Institute for Health Metrics and Evaluation, University of Washington, Seattle, Washington, USA

Correspondence to:

Dr K Bhalla, Research Scientist, Harvard University Initiative for Global Health, 104 Mt Auburn Street, Cambridge, MA, USA; kavi_bhalla@harvard.edu

Accepted 6 January 2009

\section{ABSTRACT}

Objective: To use a range of existing information sources to develop a national snapshot of the burden of road traffic injuries in one developing country-Iran.

Methods: The distribution of deaths was estimated by using data from the national death registration system, hospital admissions and outpatient visits from a timelimited hospital registry in 12 of 30 provinces, and injuries that received no institutional care using the 2000 demographic and health survey. Results were extrapolated to national annual incidence of health burden differentiated by age, sex, external cause, nature of injuries and institutional care.

Results: In 2005, 30721 Iranians died annually in road traffic crashes and over one million were injured. The death rate (44 per 100 000) is the highest of any country in the world for which reliable estimates are available. Road traffic injuries are the third leading cause of death in Iran. While young adults are at high risk in non-fatal crashes, the elderly have the highest total death rates, largely due to pedestrian crashes. While car occupants lead the death count, motorised two-wheeler riders dominate hospital admissions, outpatient visits and health burden.

Conclusions: Reliable estimates of the burden of road traffic injuries are an essential input for rational priority setting. Most low income countries are unlikely to have national injury surveillance systems for several decades. Thus national estimates of the burden of injuries should be built by collating information from all existing information sources by appropriately correcting for source specific shortcomings.

Road traffic crashes kill an estimated 1.2 million people worldwide annually (10th leading cause of death) and account for $2.6 \%$ of the total global burden of disease, measured in terms of lost years of healthy life. ${ }^{1}$ Although $85 \%$ of these deaths and $90 \%$ of the burden of disease associated with road traffic injuries are estimated to occur in low- and middle-income countries, because of the absence of reliable national data sources, estimates for most countries are based on regional extrapolations.

Because of the serious threat that road traffic crashes pose to public health, there is a substantial ongoing international effort to focus political and public attention on the problem. ${ }^{2}$ These efforts include: the release of the 2004 World Report on Road Traffic Injury Prevention ${ }^{3}$ by the World Health Organization (WHO) and the World Bank; "road safety" being designated as the theme for the 2004 World Health Day by the WHO; the recent landmark resolutions by the UN General
Assembly ${ }^{4}$; and a report aimed at adding road safety to the agenda of the G8 Summit. ${ }^{6}$ These efforts have widely acknowledged the need for generating reliable data for describing the public health burden of road traffic injuries, evaluating the impact of safety policies, and benchmarking achievements. $^{3}$ As a result, regional and global development agencies, such as the WHO, the United Nations Economic and Social Commission for Asia and the Pacific (UNESCAP), and the Pan American Health Organization (PAHO), have increased their efforts to tabulate cross-national statistics. Data collection is a critical component of the international capacity-building efforts of these agencies. Unfortunately, the process of collecting road traffic injury metrics by asking country governments to report back official transportation statistics will not produce results that can be used for meaningful planning. For instance, UNESCAP collects quantitative indicators in a standardised framework by requesting countries to report road deaths, deaths per vehicle, deaths per billion vehicle-km, non-fatal injuries and non-injury crashes, etc. ${ }^{7}$ However, most of these variables cannot be reliably estimated, even in high income countries that have extensive injury surveillance infrastructure.

Instead, international agencies need to recognise that most low- and middle-income countries will not have surveillance infrastructure with the capacity to directly report national injury metrics for several decades. Even local injury surveillance systems are of little use for national policy planning because they do not usually cover the national population. Instead, the focus should be on collating all existing information sources in a country (eg, death registers, hospital records, mortuary data, crematorium records, health surveys, crime reports), correcting for source specific shortcomings, and triangulating to a national snapshot of road traffic injuries. Since such record keeping is not usually intended for population injury surveillance, it is important to understand the limitations and biases of each data source. Often, the data will not be population representative, have low coverage and completeness, and be of poor quality with large numbers of cases coded to poorly specified causes. Thus, there is a need for validated tools that map such information sources to national indicators of road traffic injury metrics that can be used to inform national priority setting.

In this paper, we present the first step towards such an approach in a developing country-the 


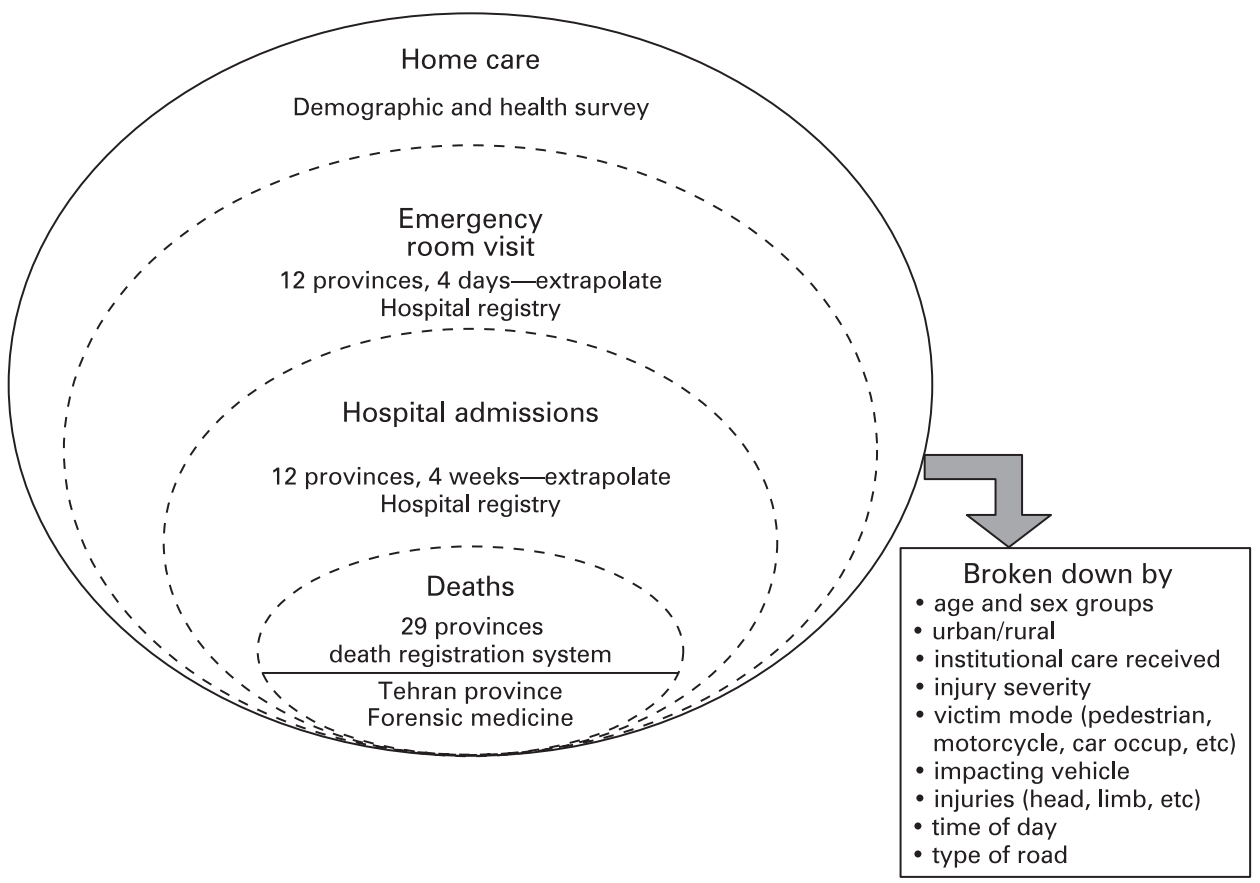

Figure 1 Developing a national snapshot of road traffic injuries from all available data sources in Iran. Source: deaths and non-fatal cases in hospital registry (12 provinces, limited time period).

Islamic Republic of Iran. We rely on death registration data, limited data collection from hospitals (admissions and outpatient visits), and a national health survey to build a comprehensive assessment of the burden of road traffic injuries in Iran differentiated by age, gender, location (urban and rural), victim's mode of transport (pedestrian, car occupant, etc), and the type of care received (hospital inpatient admissions, outpatient visits, or care at home). Although this paper only focuses on Iran, one of our primary goals is to develop a process that can be replicated in all countries.

\section{METHODS}

\section{Data sources}

Figure 1 illustrates the general strategy for estimating national burden of road traffic injuries from the various data sources in this study.

Case-level unit record data from the 2005 death registration records were obtained from the Iranian Ministry of Health and Medical Education (MOHME). The Death Registration System (DRS) collects mortality data from district health centres. In rural areas, deaths are registered in approximately 17000 health houses and reported to the district health centres. At the district level, death registration data are obtained from five sources: (1) all public and private hospitals; (2) official district cemeteries; (3) the district office of the Forensic Medicine Organization; (4) household visits by community health workers in rural areas; and (5) information from community health volunteers, clergymen and printed obituaries. In most cases, a physician provides death certification with cause of death reported in a format similar to the International Classification of Diseases 10th Revision. In 2005, which is the study year, the death registration system covered all 30 provinces except Tehran.

We obtained microdata from a hospital sample registry conducted by MOHME in 2005, which collected injury case information from all hospitals in 12 provinces in a specified time period. This data included all inpatient cases (discharged alive) during the first week of the last month of each season (4 weeks in total), and all outpatient cases during one randomly assigned day of each of these four weeks (4 days in total), amounting to a total of 2572 outpatient records and 9123 inpatient records. Data were collated from the medical records of the victim and an interview with household members of the victims. Data collected included variables that characterised the demographic and socioeconomic characteristics of victims, vehicles involved, highway types, environmental conditions, and injury and care details.

Finally, we obtained data for the nationally representative year 2000 Demographic and Health Survey (DHS), which covered 56674 urban households and 54952 rural households. We analysed responses to three consecutive questions: (1) "Has any member of your household suffered an accident during the past month?" (response categories: "yes", "no", "do not

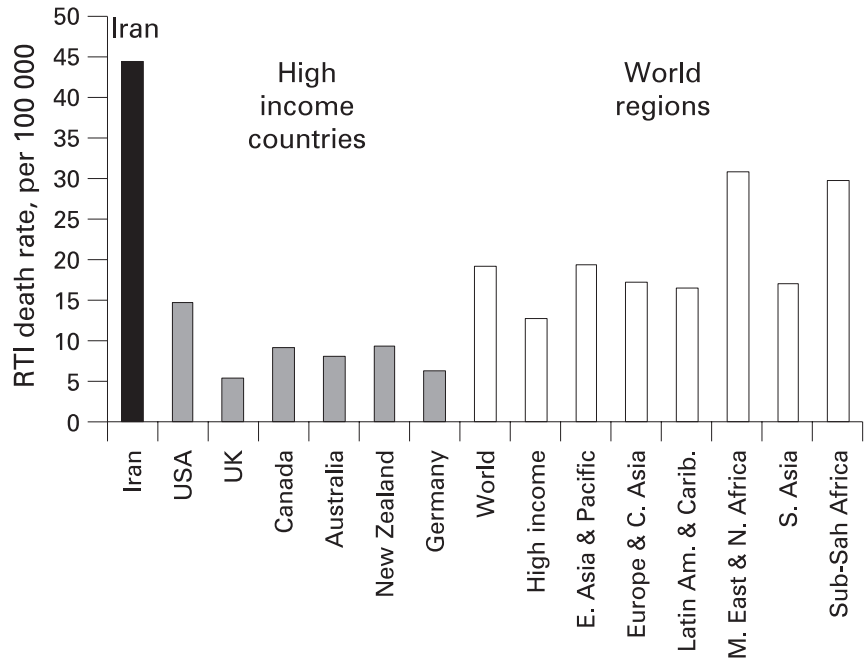

Figure 2 Road traffic injury (RTI) death rate in Iran compared with other countries and world regions. 
know"); (2) "What was the type of accident?" ("traffic accident" was one of the response categories); and (3) "What treatment did s/he receive?" (response categories: "inpatient", "outpatient", "at home", "no treatment").

\section{Definitions}

The full set of definitions are described elsewhere, ${ }^{8}$ but key definitions are summarised as follows. A road traffic crash is an event that produces injury and/or property damage, involves a vehicle in transport, and occurs on a road or while the vehicle is still in motion after running off the public highway. An injury is the reduction in functional health status due to energy exchanges that have relatively sudden discernable effects. Institutional care for injuries is considered an inpatient admission if it involves a hospital stay of over 24 hours, and an outpatient visit, if less. For road traffic deaths, the relevant ICD-10 codes were: pedestrian, V01-V04, V06-V09; bicycles, V10-V19; motorised two-wheeler, V20-V29; motorised three-wheeler, V30-V39; car/van, V40-V59; truck, V60-V69; bus, V70-V79; and other road users, V80-V86.

\section{Analysis}

We tabulated all datasets within age (11 groups), sex and residence (urban/rural) groups. The hospital inpatient and outpatient datasets were further broken down by mode of transport categories. Ill-specified cases were a substantial problem in the death registration dataset (table 1). We reassigned deaths coded to unspecified transport accidents, injuries from unspecified intent, and broader less-specified causes of death. The redistribution of cases classified to unspecified categories to the relevant specified categories was done in proportion to the number of specified cases within age, sex and urban/rural categories (ie, age-sex-urban/rural pro-rata redistribution). There were no ill-specified and unknown cases for the variables of interest in the hospital inpatient and outpatient datasets.

Table 1 Unknowns and ill-defined cases in death registration data from 29 (of 30) provinces

\begin{tabular}{lrl}
\hline & Death records & Redistributed over \\
\hline Total death records & $244341(100 \%)$ & - \\
Unknown province & $2962(1.2 \%)$ & Specified provinces \\
Unknown sex & $373(0.2 \%)$ & Specified sex \\
Unknown age & $1845(0.8 \%)$ & Specified age \\
Unknown residence (urban/rural) & $0(0.0 \%)$ & -
\end{tabular}

\section{Total transport injuries}

Unspecified transport injuries

Injuries of undetermined intent

Unspecified cause of death;

cause under investigation

Septicaemia

Mental retardation

Convulsion newborn

Senility

Sudden infant death syndrome

Convulsions not elsewhere

classified

Other ill-defined unregistered cause of death;

$\begin{aligned} 25531(10.4 \%) & - \\ 15122(6.2 \%) & \text { Specified transport mode } \\ 126(0.1 \%) & \text { All specified injuries } \\ & \text { (including transport) } \\ 9156(3.7 \%) & \text { All causes } \\ & \\ 1009(0.4 \%) & \\ 405(0.2 \%) & \\ 0(0.0 \%) & \\ 13505(5.5 \%) & \\ 122(0.0 \%) & \\ 19(0.0 \%) & \end{aligned}$

$1559(0.6 \%)$
After redistribution, transport accidents total 28221 (ie, increase of 10.5\%). This value does not include deaths in the province of Tehran (which is not covered by the death registration system).
Because the death registration data did not include the province of Tehran, we attempted generating estimates for this province using several different methods. For instance, we computed the estimates for Tehran province by applying the age-sex-urban/rural- and victim mode-specific death rates to the population of Tehran (6449 road traffic deaths). Because most of the population of Tehran province lives in the metropolitan city Tehran, we also obtained estimates by applying death rates from the next five most populous cities in Iran (Ahvaz, Esfhan, Mashhad, Shiraz and Tabriz). We found a variation of over $400 \%$, suggesting that road traffic death rates vary considerably between cities. Thus, we used an alternate data source, forensic medicine, which reported province totals of road traffic deaths in Iran that were broadly consistent with our estimates from the death registration system for the remaining 29 provinces. Since forensic medicine did not provide a further breakdown of the 2645 road traffic injury deaths in Tehran, we have assumed the age-sex- and victim mode-breakdown computed from the other 29 provinces applies to Tehran.

Because bicycles are comparatively uncommon in Iran, the Iranian death registration system records these deaths in the same category as motorised two-wheelers. We estimated that $7.5 \%$ (363 deaths) were bicycle riders based on a comparison of the incidence of bicycle mortality reported in 13 health divisions by Karkhaneh et $a l^{9}$ and the ratio of bicycle cases to motorised two-wheeler cases in the hospital admissions dataset.

For the hospital inpatient and outpatient data, we extrapolated road traffic injury rates during the time period of data collection in the 12 provinces to generate the annual estimate for the national population. Similarly, for the health survey we adjusted the monthly road traffic involvement rate to generate annual incidence estimates.

Finally, we computed burden of disease estimates using methods that were developed by the Global Burden of Disease (GBD) study. ${ }^{10}{ }^{11}$ The weights and durations were derived from the $\mathrm{GBD}^{12}$ and Victorian Burden of Disease ${ }^{13}$ studies. We used a modified method to account for multiple co-morbidities using an individual level multiplicative model for disability weights. More details of the method can be found elsewhere. ${ }^{14} 15$

We express the results using disability adjusted life years (DALYs), which represent years of life lost to premature death and years lived with a disability of specified severity and duration. DALYs are computed by adding the numbers of years of life lost (YLLs) in fatal crashes and the total years of life lost due to disabilities (YLDs) by survivors in non-fatal crashes. YLLs correspond to the number of deaths multiplied by the standard

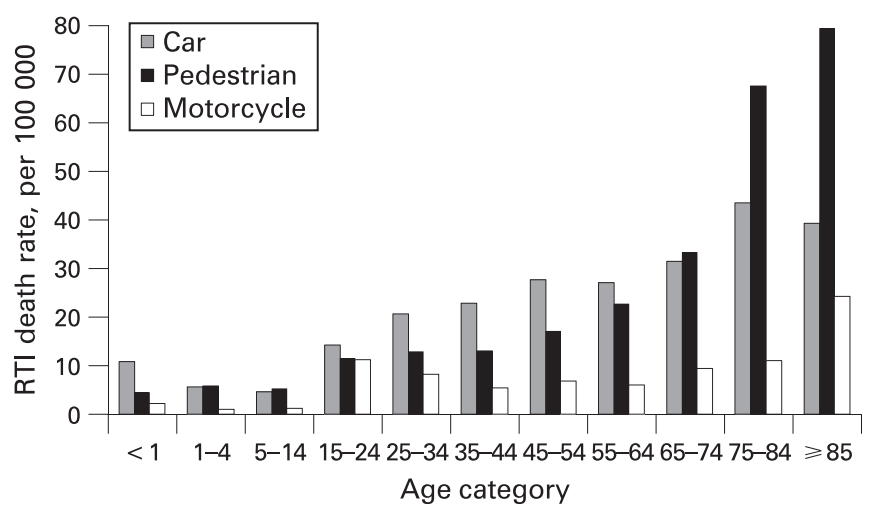

Figure 3 Age distribution of pedestrian, motorcycle rider and car occupant deaths. RTI, road traffic injury. 
Table 2 Annual road traffic crashes in Iran classified by the care they received

\begin{tabular}{|c|c|c|c|c|c|c|c|c|c|c|c|c|}
\hline \multirow[b]{2}{*}{ Age (years) } & \multicolumn{2}{|l|}{ Fatal } & \multicolumn{2}{|c|}{ Hospital inpatient } & \multicolumn{2}{|c|}{ Hospital outpatient } & \multicolumn{2}{|c|}{ Home care } & \multicolumn{2}{|l|}{ No care } & \multicolumn{2}{|l|}{ Total } \\
\hline & Cases & Rate & Cases & Rate & Cases & Rate & Cases & Rate & Cases & Rate & Cases & Rate \\
\hline$<1$ & 234 & 20 & 770 & 67 & 2134 & 186 & 0 & 0 & 0 & 0 & 3138 & 273 \\
\hline $1-4$ & 714 & 16 & 4578 & 101 & 9038 & 200 & 16305 & 770 & 7927 & 393 & 38562 & 1480 \\
\hline $5-14$ & 2031 & 14 & 23794 & 160 & 56664 & 380 & 86480 & 1106 & 55864 & 715 & 224833 & 2374 \\
\hline $15-24$ & 7797 & 45 & 98325 & 568 & 210111 & 1213 & 70237 & 944 & 55875 & 750 & 442346 & 3519 \\
\hline $25-34$ & 6057 & 53 & 50306 & 438 & 105045 & 915 & 37373 & 760 & 70284 & 1429 & 269065 & 3595 \\
\hline $35-44$ & 4196 & 54 & 27339 & 350 & 44889 & 574 & 38801 & 1067 & 55867 & 1536 & 171092 & 3580 \\
\hline $45-54$ & 3366 & 65 & 20191 & 392 & 31833 & 618 & 20461 & 880 & 30038 & 1297 & 105889 & 3252 \\
\hline $55-64$ & 2144 & 70 & 12049 & 391 & 16497 & 535 & 6411 & 395 & 8378 & 490 & 45479 & 1881 \\
\hline $65-74$ & 40 & 93 & 7191 & 297 & 10412 & 431 & 8552 & 701 & 5752 & 461 & 34147 & 1983 \\
\hline $75-84$ & 1650 & 157 & 3464 & 330 & 3733 & 356 & 5701 & 1272 & 1568 & 293 & 16116 & 2408 \\
\hline $85+$ & 293 & 185 & 335 & 212 & 951 & 601 & 0 & 0 & 0 & 0 & 1579 & 998 \\
\hline Total & 30721 & 44 & 248341 & 360 & 491307 & 711 & 290321 & 457 & 291553 & 445 & 1352244 & 2017 \\
\hline
\end{tabular}

Rate is per 100000 population.

life expectancy at the age at which the death occurs after time discounting and age-weighting which gives less weight to years lived at younger and older ages. Similarly, YLDs are estimated by multiplying the number of incident non-fatal injuries with a disability weight for the injury and the average duration (in years) of the disability resulting from the event.

\section{RESULTS}

In 2005, road traffic crashes resulted in 30721 deaths in Iran, amounting to an annual road traffic death rate of 44 per 100000 people (fig 2). Among males between the ages of 15 and 24 years, $52 \%$ of all deaths are due to road traffic crashes. Similarly, in the broader male age group of 15-44 years, 44\% of all deaths are due to road traffic crashes.

As table 2 shows, 1.06 million people (1.5\% of population) suffered injuries for which they were admitted to hospital (248 341 people), received outpatient care (491 307 people), or were treated at home (290 321 people). An additional 0.29 million people were involved in crashes that did not receive any care.

Rates of hospital inpatient admissions and outpatient visits have an age trend that is different from that of deaths. While the death rate increases steadily with age, the rate of non-fatal injuries peaks in the age group of 15-24 years and declines for higher ages. Road traffic death rates among the elderly $(\geqslant 65$

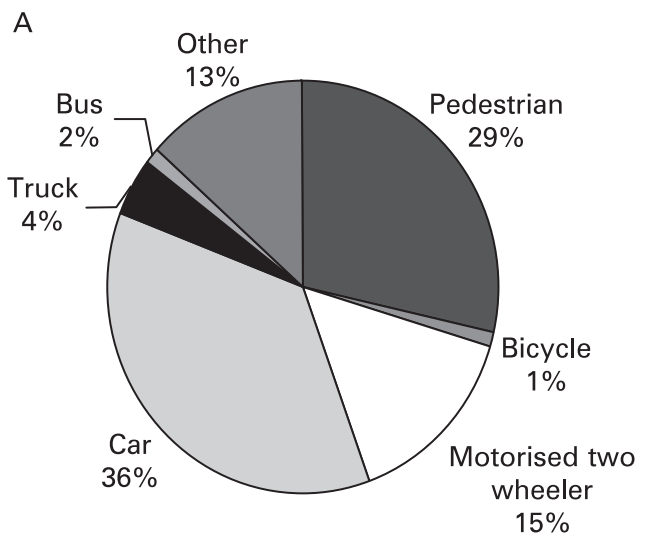

Deaths years) are 2.6 times that of the general population. However, age-specific death rates vary substantially depending on the victim's mode of transport (fig 3).

The distribution of road traffic crashes by victim's mode of transport is substantially different for deaths and non-fatal cases (fig 4). Car occupants (36\% of all deaths) comprise the largest death category, followed by pedestrians (29\%) and motorised two-wheeler riders (15\%). However, over half of all hospital admissions and outpatient visits for road traffic crashes are riders of motorised two-wheelers. Table 3 describes the ratio of hospital admissions and outpatient visits to deaths for different victim transport modes. Motorised two-wheeler crashes result in a dramatically higher number of inpatient admissions (27.8) and outpatient visits (55.8) per death than for all road traffic crashes (8.1 and 16.0 , respectively). The burden imposed by motorised two-wheeler riders on the Iranian healthcare system is not apparent from death statistics.

As recommended in the literature, ${ }^{23}{ }^{24}$ fig 5 uses multiple injury profiles to compare distribution of injuries among fatal, inpatient and outpatient cases. The injury profiles differ substantially, with multiple injuries being more common in victims that died. Head injuries dominate (39\% of all injuries) in fatalities. Among inpatient admissions, open wounds and crushing (23\%), head injuries (19\%), and lower limb injuries $(19 \%)$ occur with similar frequency. Among outpatient visits,

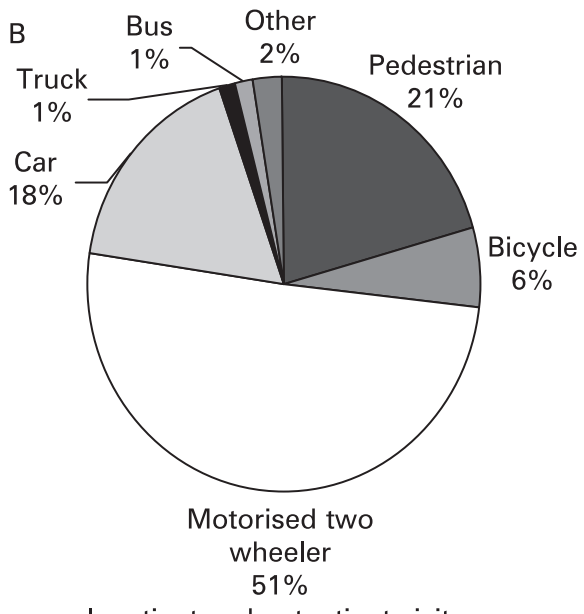

Inpatient and outpatient visits

Figure 4 Distribution of road traffic crashes by victim's mode of transport. Burden was computed for deaths, hospital admissions and outpatient visits. 
Table 3 Ratio of inpatient hospital admissions and outpatient visits to deaths

\begin{tabular}{lllcc}
\hline Sex & Victim mode & Fatal & Inpatient & Outpatient \\
\hline Both & Pedestrian & 1 & 5.7 & 11.9 \\
& Motorised two- & 1 & 27.1 & 55.8 \\
& wheeler & & & \\
& Car & 1 & 4.6 & 7.0 \\
& Truck & 1 & 2.2 & 4.9 \\
& Bus & 1 & 11.8 & 10.4 \\
& Other & 1 & 1.2 & 3.3 \\
& & & & \\
Male & Pedestrian & 1 & 4.7 & 9.9 \\
& Motorised two- & 1 & 26.4 & 53.1 \\
& wheeler & & & \\
& Car & 1 & 4.1 & 6.5 \\
& Truck & 1 & 2.4 & 5.4 \\
& Bus & 1 & 10.8 & 9.2 \\
& Other & 1 & 1.2 & 2.8 \\
& Total & 1 & 8.1 & 15.9 \\
& & & & \\
Female & Pedestrian & 1 & 9.7 & 19.8 \\
& Motorised two- & 1 & 34.9 & 95.7 \\
& wheeler & & & \\
& Car & 1 & 5.6 & 8.6 \\
& Truck & 1 & 1.0 & 1.2 \\
& Bus & 1 & 14.0 & 13.2 \\
& Other & 1 & 0.5 & 3.3 \\
& Total & 1 & 8.2 & 16.3 \\
& & & & \\
& & 1 & 8.1 & 16.0 \\
\hline
\end{tabular}

open wounds and crushing (40\%) are the most common form of injury.

Overall, 1.2 million years of healthy life-years were lost to injuries from road traffic crashes. Two-thirds of this public health burden is due to years of lives lost (YLLs) due to deaths (fig 6). Injuries to riders of two-wheelers are the leading cause of the burden of road traffic injuries ( $31 \%$ of total burden) followed by car occupants (30\%), and pedestrians (26\%).

\section{DISCUSSION}

\section{Implications for Iran}

The annual road traffic death rate (44 per 100 000) exceeds the average death rate in any region of the world for which reliable estimates of road traffic crashes are available (fig 2). Compared with other health problems faced by Iran, road traffic crashes present a significant challenge, killing more people annually than the death toll of the 2003 Bam earthquake. They are the third leading cause of death in Iran, resulting in $10.3 \%$ of all deaths, almost five times the world average (2.1\%). In addition to deaths, road traffic crashes result in a large number of nonfatal disabling injuries. Almost 0.8 million people $(1.1 \%$ of the population) seek hospital care for road traffic injuries annually, resulting in a substantial burden on Iranian healthcare institutions.

As in other countries, males are much more likely to be killed or injured in a road traffic crash than females, especially among adults and the elderly, likely reflecting gender disparities that result in lower travel among women. However, death rates are highest among the elderly despite their lower rate of involvement in non-fatal crashes. This pattern is particularly pronounced for pedestrians and thus likely reflects lower biomechanical tolerances to injuries. A similar age pattern exists in other countries (for instance, in the USA ${ }^{16}$ ), but the risk faced by the elderly is more pronounced in Iran. Clearly, road safety needs to be a priority health concern for all ages, including the elderly.

While car occupants comprise the single largest category of road users killed in road traffic crashes, motorcycle riders are the leading cause of hospital visits (primarily for lower limb injuries, open wounds and crushing) compared with other road crash victims even though there are fewer motorcycle deaths than pedestrian and car occupant deaths. Because of the disability associated with the vast number of non-fatal injuries resulting from motorcycle crashes, motorcycle riders bear the largest fraction of the burden (measures in DALYs) among all road users.

Even though the data sources used in this analysis are incomplete and have a range of data quality issues, Iran cannot afford to wait to implement safety policies. There is a critical need for the government of Iran to track the problem using all currently available information to establish national road safety priorities and implement a safety plan that embodies the best practices outlined in the 2004 World Report on Road Traffic Injury Prevention. ${ }^{3}$

\section{Implications for global road safety research}

What we recommend for Iran needs to be replicated in all countries that do not currently have a reliable mechanism for estimating the burden of road traffic injuries. Until such surveillance infrastructure is established, all existing information sources should be used to triangulate to a national snapshot
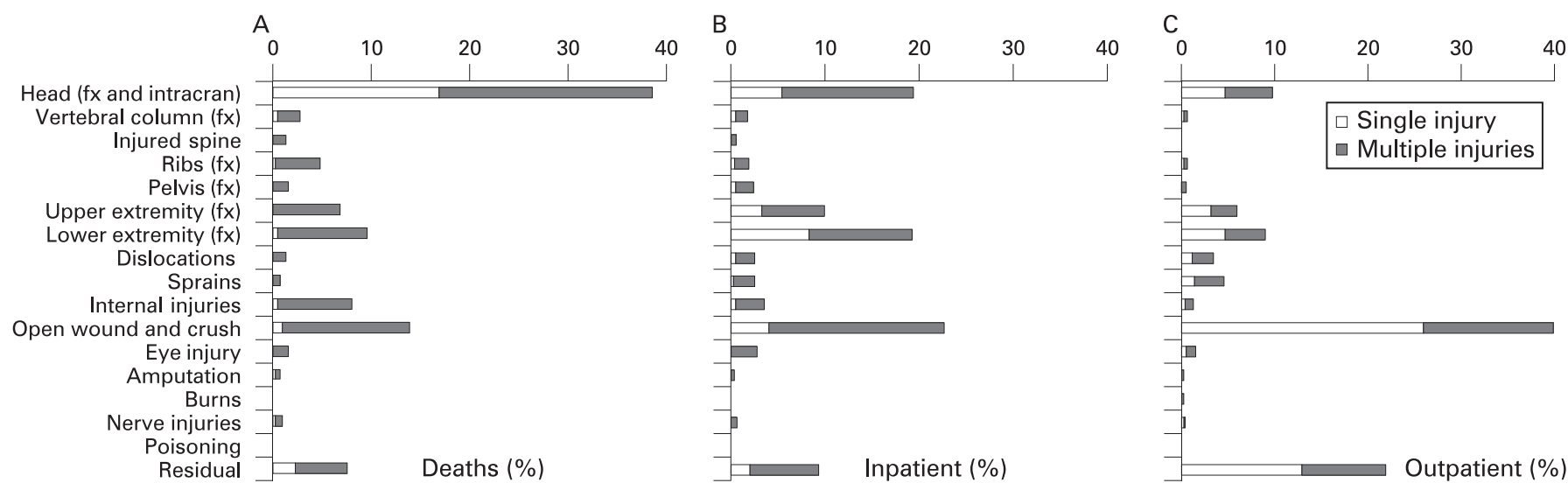

Figure 5 Multiple injury profiles for victims of road traffic crashes that resulted in (A) death, (B) inpatient hospital admission, and (C) outpatient visit. 


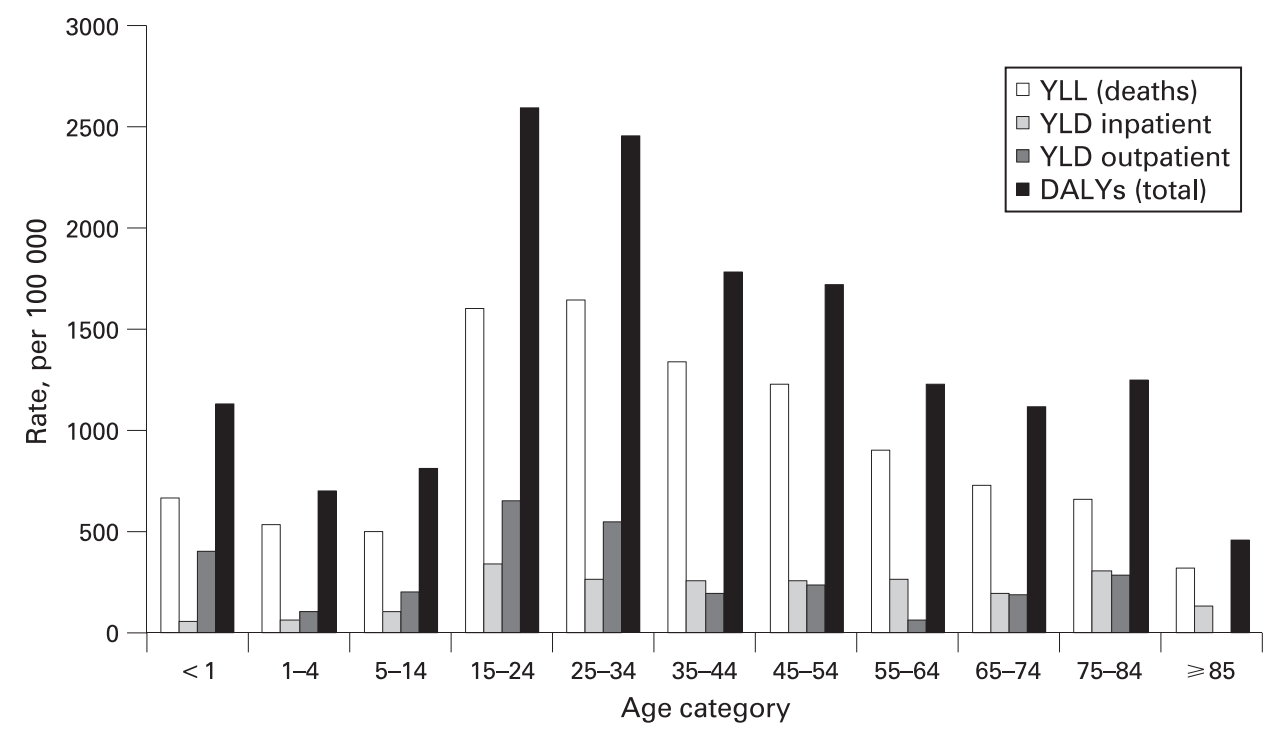

Figure 6 Public health burden of fatal road traffic crashes and non-fatal road traffic crashes that received institutional care by age. Source: Road traffic injury death rates in Iran based on our analysis of death registration data; in high income countries based on IRTAD; and in World Regions based on GBD 2002.' YLL, years of life lost; YLD, years of life lost due to disabilities; DALYs, disability adjusted life years.

of road traffic injuries. When these data sources are not originally intended for surveillance, appropriate care needs to be taken to understand and correct for source specific biases and limitations.

At present, official government statistics of road traffic deaths and injuries in most low income countries are sourced from crime reports. Although substantial underreporting of non-fatal injuries in police reports is widely acknowledged, ${ }^{17}$ even in high income countries, ${ }^{18}{ }^{19}$ it is often assumed that death counts are more reliable. In Iran, our estimate of road traffic deaths are almost seven times the number reported by the police (4441 in 2005). But in recent years, health researchers ${ }^{14}$ have increasingly used improving death registration data to firmly establish that the police death counts are too low. We expect that using alternate sources for estimating road traffic deaths in other countries will likely uncover widespread underreporting of deaths in official government statistics.

Although population representative estimates of the incidence of non-fatal injuries are analytically more difficult to

\section{Key points}

\section{What is already known on this topic}

- Reliable national statistics of road traffic injures are an essential input for defining national health priorities.

- Most developing countries will not have reliable injury surveillance systems capable of producing such estimates in the foreseeable future.

\section{What this study adds}

- National estimates of road traffic injuries can be built by collating and extrapolating information from all available data sources. We show this process for Iran.

- National estimates should be derived for both fatal and nonfatal injuries because the pattern of non-fatal injuries can be very different from those of fatal injuries. derive than death estimates, these can reveal a different set of safety problems. In Iran, the true magnitude of injuries to motorcycle riders, who account for more than half of all nonfatal road traffic victims, was only evident from an analysis of hospital records. Analysis of deaths alone, as is usually the case, would have placed motorised two-wheelers behind car occupants and pedestrians in terms of burden. Estimating incidence from hospital records is usually difficult because they are not a population representative sample. While having access to all hospital records in a region allowed us to compute population estimates of incidence, recent methodological advances ${ }^{2021}$ are helping increase the use hospital records in such epidemiological research. Similarly there is a need for developing methods to account for poor data quality. ${ }^{22}$

The international injury research community needs to pool their expertise to develop and validate the analytical tools that will allow such estimates to be reliably built from the vast range of untapped information sources that already exist. Such a toolkit would be a global public good that would enable nations to plan a safe and sustainable future for all.

Acknowledgements: We are grateful to the Iranian Ministry of Health and Medical Education (MOHME) and its divisions, in particular the Deputy of Health, the Network Development and Health Promotion Center, Dr Esmaeel Motlagh and Dr Nahid Jafari for providing access to datasets.

Funding: This work was funded by a grant from the World Bank Global Road Safety Facility. The funding agency did not play any role in the study design, analysis and interpretation of data, in the writing of the report, or in the decision to submit the paper for publication.

Competing interests: None.

\section{REFERENCES}

1. World Health Organization. Global burden of disease-2002. http://www.who.int/ topics/global burden of disease/en/ (accessed 15 April 2009).

2. Ameratunga $\mathbf{S}$, Hijar $\bar{M}$, Norton R. Road-traffic injuries: confronting disparities to address a global-health problem. Lancet 2006;367:1533-40.

3. Peden MM. World report on road traffic injury prevention. Geneva: World Health Organization, 2004.

4. United Nations General Assembly. The global road safety crisis: progress on the implementation of General Assembly Resolution 58/289. A/60/181. 2005.

5. United Nations General Assembly. Improving Global Road Safety. A/62/L.43. 2008. 
6. Commission for Global Road Safety. Make roads safe: a new priority for sustainable development. http://www.makeroadssafe.org/ (accessed 15 April 2009)

7. United Nations Economic and Social Commission for Asia and the Pacific Road safety in Asia and the Pacific. http://www.unescap.org/ttdw/roadsafety/index. html (accessed 15 April 2009).

8. Bhalla K. Road traffic injury metrics-recommended definitions. Cambridge, MA: Harvard University Initiative for Global Health Road Traffic Injury Metrics Group, 2007.

9. Karkhaneh $\mathbf{M}$, Naghavi $\mathbf{M}$, Rowe $\mathbf{B H}$, et al. Epidemiology of bicycle injuries in 13 health divisions, Islamic Republic of Iran 2003. Accid Anal Prev 2008;40:192-9.

10. Murray CJL, Lopez AD. The global burden of disease: a comprehensive assessment of mortality and disability from diseases, injuries and risk factors in 1990 and projected to 2020. Global Burden of Disease and Injury Series. Cambridge, MA: Harvard University Press, 1999:1.

11. Murray CJ, Lopez AD. Mortality by cause for eight regions of the world: Global Burden of Disease Study. Lancet 1997:349:1269-76.

12. Murray CJL, Lopez AD. The global burden of disease: a comprehensive assessment of mortality and disability from diseases, injuries and risk factors in 1990 and projected to 2020. Global Burden of Disease and Injury Series. Cambridge, MA Harvard University Press, 1999;1.

13. Vos T, Begg S. The Victorian Burden of Disease Study: Morbidity. Melbourne: Department of Human Services, 2000.

14. Naghavi M, Abolhani F, Moradi M, et al. National burden of disease and injury in Iran-2003. Tehran: Ministry of Health and Medical Education of Iran, 2007.
15. Begg S, Vos T, Barker B, et al. The burden of disease and injury in Australia, 2003 Canberra: Australian Institute of Health and Welfare, 2007.

16. Finkelstein EA, Corso PS, Miller TR. The incidence and economic burden of injuries in the United States. New York: Oxford University Press, 2006

17. Aeron-Thomas A. Underreporting of road traffic casualties in low income countries. PR/INT/199/00. Transportation Research Laboratory, 2000.

18. Amoros E, Martin JL, Laumon B. Estimating non-fatal road casualties in a large French county, using the capture-recapture method. Accid Anal Prev 2007;39:483-90.

19. Elvik R, Mysen AB. Incomplete accident reporting: meta-analysis of studies made in 13 countries. Transportation Research Record 1999:1665:133-40.

20. Murray CJL, Lopez AD, Barofsky JT, et al. Estimating population cause-specific mortality fractions from in-hospital mortality: validation of a new method. PLoS Med 2007; 4:e326.

21. Alexandrescu R, O'Brien SJ, Lyons RA, et al. A proposed approach in defining population-based rates of major injury from a trauma registry dataset: delineation of hospital catchment areas (I). BMC Health Services Research 2008;8:80.

22. Bhalla K, Shahraz S, Naghavi M, et al. Estimating the distribution of external causes in hospital data from injury diagnosis. Accident Analysis and Prevention 2008; 40:1822-9.

23. Aharonson-Daniel L, Boyko V, Ziv A, et al. A new approach to the analysis of multiple injuries using data from a national trauma registry. Inj Prev 2003:9:156-62.

24. Aharonson-Daniel L, Giveon A, Peleg K. Gaps in injury statistics: multiple injury profiles reveal them and provide a comprehensive account. Inj Prev 2005:11:197-200.

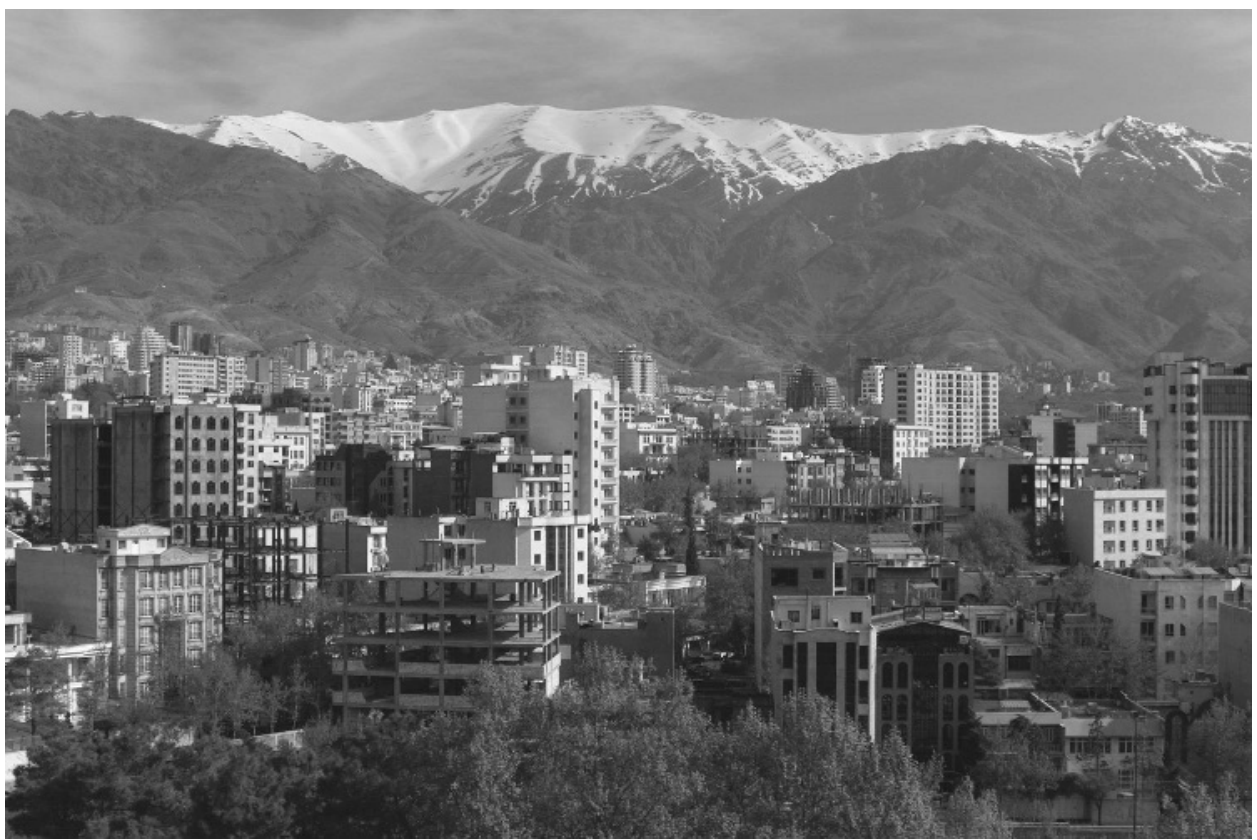

